\title{
SERS imaging-based aptasensor for ultrasensitive and reproducible detection of influenza virus A
}

\author{
Hao Chen ${ }^{\text {a, } 1}$, Sung-Gyu Park ${ }^{\text {b, }}$, Namhyun Choi ${ }^{\text {a }}$, Joung-Il Moon ${ }^{\text {a }}$, Hajun Dang ${ }^{\text {a }}$, Anupam Das ${ }^{\text {a }}$, \\ Seunghun Lee ${ }^{\mathrm{b}}$, Do-Geun Kim ${ }^{\mathrm{b}}$, Lingxin Chen ${ }^{\mathrm{c}, * *}$, Jaebum Choo ${ }^{\mathrm{a},}$

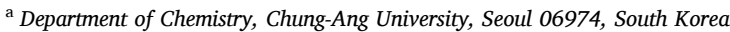 \\ ${ }^{\mathrm{b}}$ Advanced Nano-Surface Department, Korea Institute of Materials Science (KIMS), Changwon, 51508, South Korea \\ ${ }^{c}$ CAS Key Laboratory of Coastal Environmental Processes and Ecological Remediation, Institute of Coastal Zone Research, Chinese Academy of Sciences, Yantai, 264003, \\ China
}

\section{A R T I C L E I N F O}

\section{Keywords:}

Surface-enhanced Raman scattering (SERS)

Aptasensor

SERS imaging sensor

Influenza A virus

SERS-based assay

\begin{abstract}
A B S T R A C T
Surface-enhanced Raman scattering (SERS)-based aptasensors display high sensitivity for influenza A/H1N1 virus detection but improved signal reproducibility is required. Therefore, in this study, we fabricated a threedimensional (3D) nano-popcorn plasmonic substrate using the surface energy difference between a perfluorodecanethiol (PFDT) spacer and the Au layer. This energy difference led to Au nanoparticle self-assembly; neighboring nanoparticles then created multiple hotspots on the substrate. The localized surface plasmon effects at the hot spots dramatically enhanced the incident field. Quantitative evaluation of A/H1N1 virus was achieved using the decrease of Raman peak intensity resulting from the release of Cy3-labeled aptamer DNAs from nanopopcorn substrate surfaces via the interaction between the aptamer DNA and A/H1N1 virus. The use of a Raman imaging technique involving the fast mapping of all pixel points enabled the reproducible quantification of A/ H1N1 virus on nano-popcorn substrates. Average ensemble effects obtained by averaging all randomly distributed hot spots mapped on the substrate made it possible to reliably quantify target viruses. The SERS-based imaging aptasensor platform proposed in this work overcomes the issues inherent in conventional approaches (the time-consuming and labor-intensiveness of RT-PCR and low sensitivity and quantitative analysis limits of lateral flow assay kits). Our SERS-based assay for detecting A/H1N1 virus had an estimated limit of detection of $97 \mathrm{PFU} \mathrm{mL} \mathrm{m}^{-1}$ (approximately three orders of magnitude more sensitive than that determined by the enzymelinked immunosorbent assay) and the approximate assay time was estimated to be 20 min. Thus, this approach provides an ultrasensitive, reliable platform for detecting viral pathogens.
\end{abstract}

\section{Introduction}

Influenza viruses can spread rapidly around the globe and cause significant morbidity and mortality, especially in children and the elderly (Blut, 2009; Dawood et al., 2009; Rewar et al., 2015). Therefore, a sensitive, specific, and rapid detection technique for pandemic-causing influenza viruses is essential for controlling outbreaks. Such a technique would enable early-stage diagnosis, facilitate antiviral treatment initiation, and provide infection surveillance, particularly for those at high risk for influenza-related complications. Three types of influenza viruses affect humans: Types A, B, and C. The influenza A virus can be further subdivided into several different serotypes based on antibody response to the virus. Of these, the influenza A virus subtype H1N1 (A/H1N1) has been responsible for major public health concerns of international importance; it caused the Spanish flu in 1918 and the swine flu in 2009 (Chartrand et al., 2012; Hawkes et al., 2010).

Currently, polymerase chain reaction (PCR)-based detection is the most reliable tool for clinical diagnosis of influenza A virus in respiratory specimens (Merckx et al., 2017; Vos et al., 2019). Not only can PCR-based assays detect influenza A virus with high sensitivity, but they can also discriminate among different influenza A virus subtypes using specific primers and probes, have an accuracy of approximately $97 \%$,

\footnotetext{
* Corresponding author.

$* *$ Corresponding author.

E-mail addresses: lxchen@yic.ac.cn (L. Chen), jbchoo@cau.ac.kr (J. Choo).

1 These authors contributed equally to this work.
} 
and have a limit of detection (LOD) ranging from 0.1 to $100 \mathrm{PFU} \mathrm{mL}-1$ for influenza A virus (Carr et al., 2009; Panning et al., 2012; Whiley et al., 2009). Nevertheless, PCR-based assays are expensive, require both complicated procedures and specially trained technicians, and require a few hours to provide diagnostic results. Therefore, PCR-based methods are not ideal for a routine health care setting.

Several different types of detection methods have been recently developed to meet the urgent need for the rapid and sensitive detection of clinically relevant viruses, (Chen et al., 2016; Lim et al., 2016; Xu et al., 2017; Yi et al., 2015; Zhang et al., 2015; Zhou et al., 2014). Of these, the surface-enhanced Raman scattering (SERS) detection method is considered a strong potential candidate because of its ultrasensitive detection capability (Chung et al., 2015; Shanmukh et al., 2006; Shao et al., 2014; Sun et al., 2017; Wu et al., 2020). However, despite the development of many different SERS substrate types for SERS-based bioassays, the reliable quantitative analysis of target molecules has been impeded by the poor reproducibility of SERS signals caused by the random and heterogeneous distribution of hot junctions over the plasmonic substrates (Jiang et al., 2012; Tao et al., 2003; Willets and Van Duyne, 2007). Consequently, Raman mapping methods to accurately quantify specific target molecules on SERS substrates have been developed to resolve this issue (Catala et al., 2016; Lee et al., 2010; Yang et al., 2013; Zhu et al., 2016). Obtaining Raman images through the continuous mapping of a characteristic Raman peak enables the measurement of an average ensemble effect of SERS intensities obtained from randomly distributed hot junctions (Ko et al., 2018; Lee et al., 2014a; Park et al., 2019; Tabakman et al., 2011; Wang et al., 2018). When A/H1N1 viruses are added onto the substrate, A/H1N1 target recognition induces conformational changes of the aptamers (Hasegawa et al., 2016; Ku et al., 2015; Ruscito; DeRosa, 2016; Zhang et al., 2019), and the aptamer and A/H1N1 coupling leads to a gradual decrease in Raman signal intensities with the increase in A/H1N1 concentration.

This study fabricated a 3D nano-popcorn substrate to increase the reproducibility of the SERS-based assay and enable the ultrasensitive and reliable analysis of the influenza $\mathrm{A} / \mathrm{H} 1 \mathrm{~N} 1$ virus. Cy3-labeled aptamer probes were hybridized with thiolated capture DNAs immobilized on the 3D nano-popcorn substrate to create a strong Raman signal. This SERS-based aptasensor incorporating the aptamer-embedded nanopopcorn plasmonic substrate provides new insights for the ultrasensitive and reliable detection of viral pathogens.

\section{Materials and methods}

\subsection{Materials and reagents}

Tetrahydrofuran (99\%), 6-mercapto-1-hexanol (MCH), tris (2-carboxyethyl) phosphine hydrochloride (TCEP), and saline-sodium citrate (SSC) buffer (pH 7.0) were purchased from Sigma-Aldrich (St. Louis, MO, USA). Phosphate-buffered saline (PBS) $(10 \times, \mathrm{pH} 7.4)$ and malachite green isothiocyanate (MGITC) were purchased from Invitrogen Corporation (Carlsbad, CA, USA). Aptamer probes and capture DNA oligonucleotides were purchased from Integrated DNA Technologies, Inc. (Coralville, IA, USA); the sequences were as follows: aptamer probe, 5'-Cy3/GGG TTT GGG TTG GGT TGG GTT TTT GGG TTT GGG TTG GGT TGG GAA AAA-3'; capture DNA, 5' -ACC CAA CCC AAA CCC- $\left(\mathrm{CH}_{2} \mathrm{O}\right)_{3}$ $\left(\mathrm{CH}_{2}\right)_{3}-\mathrm{SH}-3^{\prime}$. Influenza A viruses (H1N1, H3N2) were provided by the Centers for Disease Control \& Prevention of Korea. Ultrapure water (18 $\mathrm{M} \Omega \mathrm{cm}^{-1}$ ) was obtained from a Milli-Q water purification system (Billerica, MA, USA). All chemicals used in this study were of analytical reagent grade.

\subsection{Instrumentation}

Scanning electronic microscope (SEM) images were taken using an FE-SEM instrument (SIGMA, Carl Zeiss, Germany) at an accelerating voltage of $5 \mathrm{kV}$. A JEOL TEM 2100F (JEOL, Tokyo, Japan) was used to obtain high-magnification transmittance electronic microscope (TEM) images at an accelerating voltage of $200 \mathrm{kV}$. Raman spectra and mapping images were acquired using an inVia Renishaw Raman microscope system (Renishaw, New Mills, UK). A Melles Griot He-Ne laser was used as the excitation source at a wavelength of $632.8 \mathrm{~nm}$. The Rayleigh line was removed using a holographic notch filter located in the collection path. Raman scattering signals were collected using a charge-coupled device camera with high-resolution grating (1800 grooves $\left.\mathrm{mm}^{-1}\right)$ and measured by focusing a laser spot using a $20 \times$ (NA 0.4) objective lens with a diffraction limit of $\sim 0.8 \mu \mathrm{m}$. Baseline correction of each Raman spectrum was performed using Renishaw WIRE 4.0 software. A polynomial algorithm (polynomial order $=11$ ) was applied to all points in each spectrum and the baseline was corrected as zero.

\subsection{Fabrication of 3D plasmonic nano-popcorn substrates}

A $125-\mu \mathrm{m}$-thick polyethylene naphthalate (PEN) polymer substrate (Dupont) was used after its protective film was removed. A polymer nano-dimple pattern was fabricated on the PEN film by $\mathrm{O}_{2}$ ion beam bombardment in a linear moving substrate. A linear ion source was used to generate linear $\mathrm{O}_{2}$ ion beams with a $300-\mathrm{mm}$ width (Lee et al., $2014 \mathrm{~b}$ ). The vacuum process chamber pressure was 0.9 mTorr with an $\mathrm{O}_{2}$ flow rate of $70 \mathrm{sccm}$. The PEN substrates were subjected to 60 scans by reciprocating motion at a linear moving speed of $10 \mathrm{~mm} \mathrm{~s}^{-1}$. Using a Faraday cup, which reduces secondary electron emissions by magnetic fields, the ion dose per scan was $2.3 \pm 0.2 \times 10^{15} \mathrm{~cm}^{-2}$. The mean ion energy was $700 \pm 70 \mathrm{eV}$ measured with an ion energy analyzer (Böhm and Perrin, 1993). A 100-nm-thick Au layer was directly deposited onto the PEN nano-dimples at a deposition rate of $2.0 \AA \mathrm{s}^{-1}$ using a thermal evaporation system (LAT, South Korea). The base pressure of the chamber was $9.6 \times 10^{-6}$ Torr. The prepared Au/PEN nano-dimple substrates were then treated with $97 \%$ perfluorodecanethiol (PFDT; Sigma-Aldrich, St. Louis, MO, USA). Specifically, $10 \mu \mathrm{L}$ of 97\% PFDT solution was poured into a glass Petri dish; then, the Petri dish lid was attached to the $\mathrm{Au} / \mathrm{PEN}$ nano-dimple substrate and the lid was closed for $2 \mathrm{~h}$ (Park et al., 2019). Next, an 80-nm-thick Au layer was deposited onto the PFDT-treated Au/PEN nano-dimple substrate at a deposition rate of $0.3 \AA \mathrm{s}^{-1}$ via a thermal evaporation process (LAT, Korea). The base pressure of the chamber was $9.6 \times 10^{-6}$ Torr. The deposition rate was monitored using a quartz crystal microbalance.

\subsection{Surface modification of $3 D$ nano-popcorn plasmonic substrates}

The nano-popcorn substrate surface was repeatedly cleaned with THF and deionized water. Capture DNAs were treated with a stable reducing agent (TCEP) at a $\mathrm{pH}$ of 4 for $1 \mathrm{~h}$ to activate the $\mathrm{SH}$ groups at the DNA sequence terminals. PBS solution was added to adjust the $\mathrm{pH}$ value to 7 . The substrate was then incubated in the prepared capture DNA solution for $2 \mathrm{~h}$, followed by immersion in $2 \mathrm{mM} \mathrm{MCH}$ solution for $2 \mathrm{~h}$ at room temperature; then, it was washed twice with a washing buffer. After functionalization, the capture DNAs on the substrate were hybridized with aptamer probes in $2 \times$ SSC buffer at $37{ }^{\circ} \mathrm{C}$ in a humid chamber for $2 \mathrm{~h}$, rinsed with washing buffer to remove non-hybridized aptamer probes, and dried with $\mathrm{N}_{2}$. Before the hybridization with capture DNAs, the aptamer probes were heated to $70{ }^{\circ} \mathrm{C}$ for $5 \mathrm{~min}$ to unfold their strain and cooled to $25^{\circ} \mathrm{C}$ for 10 min to keep the DNAs in unfolded forms. The capture DNA and aptamer DNA concentrations were $1 \mu \mathrm{M}$ and $1.25 \mu \mathrm{M}$, respectively.

\subsection{SERS-based aptasensor for A/H1N1 virus assay}

For the $\mathrm{A} / \mathrm{H} 1 \mathrm{~N} 1$ virus assay, $3 \mu \mathrm{L}$ of sample solution containing various concentrations of target $\mathrm{A} / \mathrm{H} 1 \mathrm{~N} 1$ virus was dropped on the surface of 3D nano-popcorn plasmonic substrates, followed by incubation for $10 \mathrm{~min}$ in a humid chamber at $37^{\circ} \mathrm{C}$. Substrates were quickly rinsed with water to prevent salt aggregation on the dried substrates. 
SERS mapping images were acquired using an inVia Renishaw Raman microscope system after the substrate dried. SERS mapping images were obtained using a point mapping method with a $20 \times$ magnification $(0.40$ NA) dry objective lens. A computer-controlled $x y$ translation stage was used to acquire a total of 625 Raman spectra with a step size of $2 \mu \mathrm{m}$ in both dimensions. All spectra in this process were obtained with an exposure time of $1 \mathrm{~s}$ and using a laser power of $0.4 \mathrm{~mW}$ after $10 \% \mathrm{ND}$ filter.

Raman reporter solution ( $10^{-6} \mathrm{M}$ MGITC) was dropped onto the substrates and their Raman mapping images were obtained using the Raman peak intensity variation at $1615 \mathrm{~cm}^{-1}$ to compare SERS performance between the nano-dimple and nano-popcorn substrates.

\subsection{ELISA for A/H1N1 virus assay}

A commercially available sandwich enzyme-linked immunosorbent assay (ELISA) kit (Influenza A Virus Nucleoprotein Antigen ELISA Kit, DEIA-CL036; Creative Diagnostics, USA) was used for A/H1N1 virus detection to compare the assay results with those measured using the SERS-based aptasensor. Assays were performed in accordance with the manufacturer's instructions. Briefly, the A/H1N1 virus solution was serially diluted 10 -fold in PBS and $200 \mu \mathrm{L}$ of each dilution was mixed with $50 \mu \mathrm{L}$ of the sample preparation reagent. One hundred microliters of each A/H1N1 virus dilution was added to the antibody-coated plate, followed by incubation for $30 \mathrm{~min}$ at room temperature on a plate shaker. Then, $100 \mu \mathrm{L}$ of influenza A/H1N1 virus detection antibody was dispensed with an equal volume of sample mixture, followed by incubation for $45 \mathrm{~min}$. After washing the plate six times with washing buffers, the sample was reacted with chromogen $(100 \mu \mathrm{L})$ for another 10 min for color development. The reaction was stopped by adding $100 \mu \mathrm{L}$ of stop solution. The optical density (OD) at $450 \mathrm{~nm}$ was measured using a microplate reader (Synergy H1 Hybrid Multi-Mode Reader, BioTek, USA). Assays were carried out independently in triplicate.

\section{Results and discussion}

\subsection{Fabrication and characterization of the 3D nano-popcorn substrate}

Fabrication of the novel 3D nano-popcorn substrate used for the SERS imaging-based A/H1N1 virus assay in this study included two sequential gold coating steps as shown in Fig. 1A. Both coating steps could be tuned to achieve different film coat properties. First, PEN surfaces were treated with $\mathrm{O}_{2}$ ion beams to generate a high areal density of polymer nano-dimple structures. Au was directly deposited onto this PEN nano-dimple substrate by thermal evaporation to fabricate a 100 $\mathrm{nm}$-thick single layer of gold nano-dimple structures. Au layer was conformally deposited on inclined surface and sharp heads of the PEN nano-dimples during the Au deposition process (Fig. S1). Au/PEN nanodimple substrates were subsequently treated with PFDT solution. The self-assembled PFDT monolayer worked as a space layer to reduce its surface energy. Instead of forming another continuous Au layer, the energy difference between Au and PFDT surfaces led to the surface diffusion of Au nanoparticles (Park et al., 2019). Consequently, Au deposited on the dimple heads formed nanoparticles, which in turn induced hot spot self-assembly during the evaporation process. Spherical AuNPs were homogeneously deposited on the surface of nano-dimple substrates and finally formed nano-popcorn substrates (Fig. 1A and Fig. S2). With the deposition of a 20-nm-thick Au layer,
A

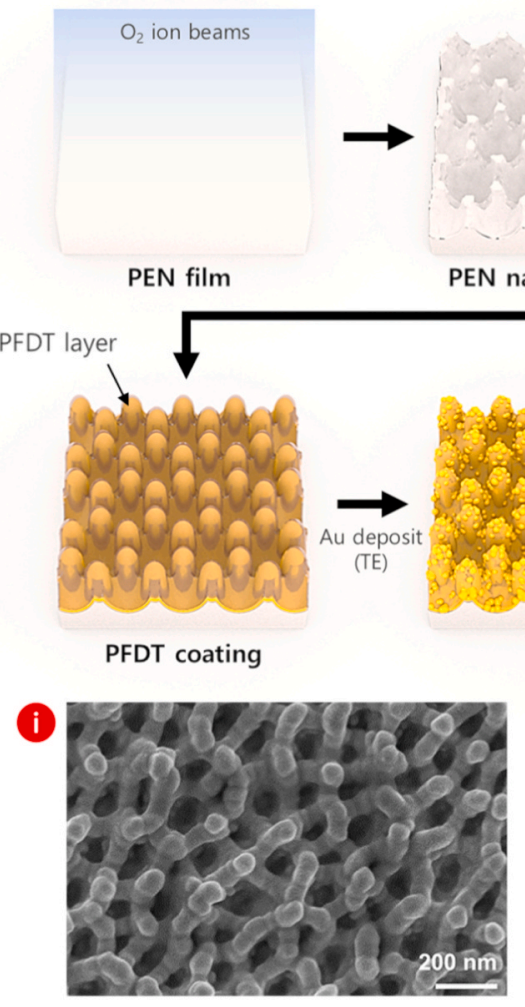

*TE: thermal evaporation

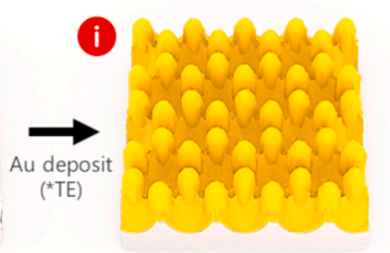

Au nano-dimple

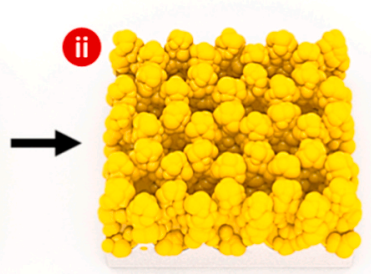

Au nano-popcorn
B

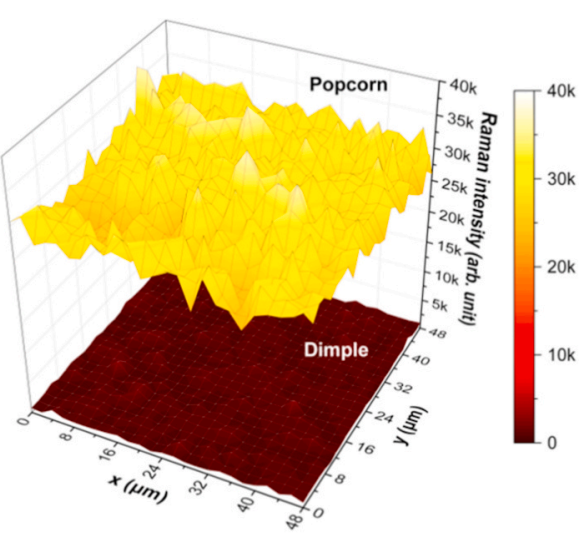

C

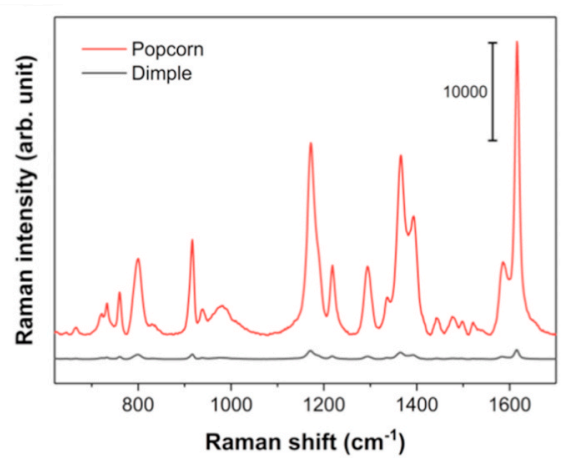

Fig. 1. Evaluation of nano-dimple and nano-popcorn structures. (A) Sequential process for 3D nano-popcorn plasmonic substrate fabrication including two gold coating steps: The 100-nm-thick gold single layer nano-dimple structures (i) and AuNP-deposited nano-popcorn structures (ii). Bottom: SEM images of nano-dimple (i) and nano-popcorn (ii) structures. (B) Raman mapping images of $10^{-6} \mathrm{M}$ MGITC at $1615 \mathrm{~cm}^{-1}$ for the nano-popcorn (yellow, top) and nano-dimple (dark red, bottom) substrates. Scanned $2 \mu \mathrm{m} \times 2 \mu \mathrm{m}$ steps over a $50 \mu \mathrm{m}$ ( $x$-axis) by $50 \mu \mathrm{m}$ (y-axis) range for a total of 625 pixels. The scale bar on the right shows the color coding used to depict Raman intensity. (C) Average Raman spectra of 625 pixels for nano-popcorn and nano-dimple substrates. (For interpretation of the references to color in this figure legend, the reader is referred to the Web version of this article.) 
AuNPs were compactly covered the entire top surface and sidewalls of the PFDT-coated Au nano-dimples. Even when the deposition thickness reached $80 \mathrm{~nm}$, the Au NPs were still spaced apart and exhibited a mean diameter of $\sim 64 \mathrm{~nm}$ (Fig. S2). This process decreased the gap distance between AuNPs and greatly enhanced local plasmonic field coupling at the longer wavelengths of the visible range (Fig. S2). Therefore, the Raman intensity of the nano-popcorn substrate was much stronger than that of the nano-dimple substrate since it retained the nanogaps necessary for hot junction formation. Raman mapping images obtained using the Raman peak intensity variation at $1615 \mathrm{~cm}^{-1}$ with an excitation laser wavelength of $632.8 \mathrm{~nm}$ compared the SERS performance between the nano-dimple and nano-popcorn substrates (Fig. 1B). The SERS mapping images showed a clear difference between the Raman responses of the two different nano-substrates. The $x-y$ translational stage used for scanning $2 \mu \mathrm{m} \times 2 \mu \mathrm{m}$ steps over a $50 \mu \mathrm{m}$ ( $x$-axis) and $50 \mu \mathrm{m}$ ( $y$-axis) range by computer control yielded 625-pixel ( 1 pixel $=2 \mu \mathrm{m} \times$ $2 \mu \mathrm{m}$ ) Raman mapping images for the square area. Total area of the nano-popcorn substrate was $5 \times 5 \mathrm{~mm}^{2}$ as shown in Scheme 1 . The accumulation number and exposure time for each pixel were 1 and $1 \mathrm{~s}$, respectively. The average SERS signal intensity of MGITC at $1615 \mathrm{~cm}^{-1}$ using the nano-popcorn substrate was approximately 30 times stronger than that of the nano-dimple substrate (Fig. 1C). This demonstrated that AuNPs formed on nano-dimple substrate generated multiple hot spots at the junctions between AuNPs and the nano-dimple surfaces, as well as between the AuNPs themselves. As a result, the nano-popcorn substrate provided a highly sensitive SERS-based detection platform.

A good SERS substrate should enable good signal reproducibility as well as a strong enhancement capability. Thus, to confirm their feasibility for bioassay applications, the reproducibility of six different $\mathrm{Au}$ nano-popcorn substrates ( $5 \mathrm{~mm} \times 5 \mathrm{~mm}$ size) were tested by dropping 3 $\mu \mathrm{L}$ of MGITC solution $\left(10^{-6} \mathrm{M}\right)$ onto six different nano-popcorn substrates (Fig. 2). The uniform light-yellow distribution for all substrates demonstrated the consistent substrate-to-substrate reproducibility.
According to our statistical analysis, the relative standard deviation (RSD) for the six substrates was as low as 6.04\% (Fig. 2D); this low RSD value indicated that the substrate-to-substrate variation in SERS intensity was very low. Based on these experimental data, Au nanopopcorn substrates exhibited very good reproducibility (see Fig. 2A).

The average enhancement factor for the 3D nano-popcorn substrate was determined using the Raman data shown in Fig. S3. Three microliters of $1 \times 10^{-10} \mathrm{M}$ MGITC solution was dropped onto the nanopopcorn substrate surface and the droplet was allowed to evaporate. From the center of the dried area, its Raman intensity (ISERS) was measured with a $20 \times$ objective lens. To establish the reference value ( $\mathrm{I}_{\mathrm{Ref}}$ ), $1 \mu \mathrm{L}$ of $10^{-3} \mathrm{M}$ MGITC was dropped on a 2D glass substrate surface and the droplet was allowed to evaporate. By measuring the Raman intensity under the same conditions, the enhancement factor was determined to be $7.18 \times 10^{7}$ for the 3D nano-popcorn substrate.

\subsection{SERS imaging-based assay for the detection of A/H1N1 virus using 3D nano-popcorn substrates}

3D nano-popcorn substrate was used as a SERS imaging platform for highly sensitive A/H1N1 virus detection. Scheme 1 demonstrates the sequential $\mathrm{A} / \mathrm{H} 1 \mathrm{~N} 1$ virus sensing strategy process using aptamerembedded nano-popcorn substrates. First, capture DNAs were immobilized onto the gold surface of nano-popcorn substrates via Au-S chemical bonds. Then, the capture DNA-immobilized SERS substrates were incubated with Cy3-labeled aptamer DNAs that can selectively and efficiently recognize A/H1N1 virus. Cy3-labeled aptamer DNAs were hybridized with capture DNAs immobilized on 3D substrates. When a large amount of Cy3-labeled aptamers were bound to 3D nano-popcorn substrates through hybridization with capture DNAs, it triggered Raman signal enhancement because the Cy3 (Raman reporter) at the $5^{\prime}$-end of the aptamer DNA was close to the 3D nano-popcorn substrate surface. Consequently, aptamer DNAs formed a confined 3D plasmonic field,
A

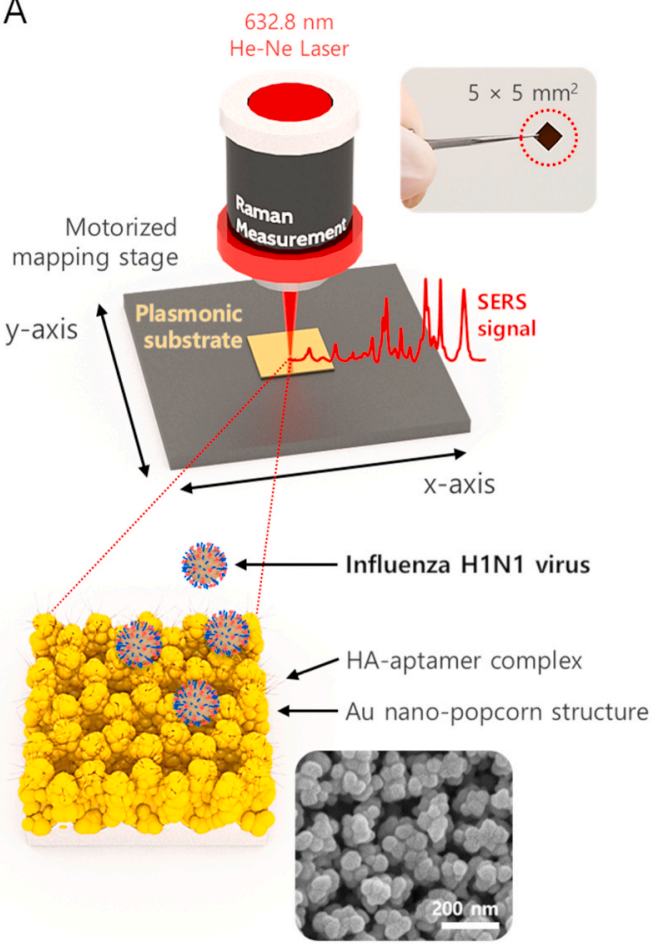

B
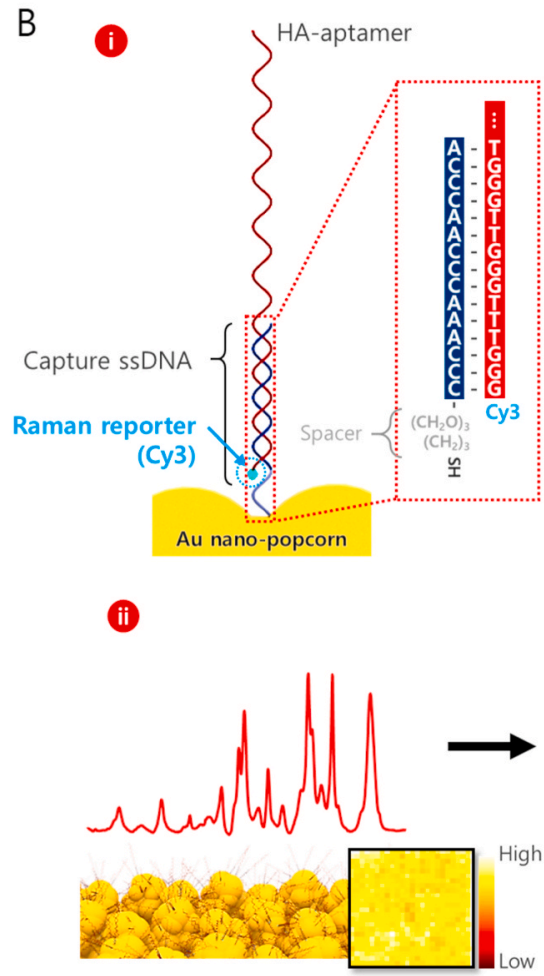

C 1

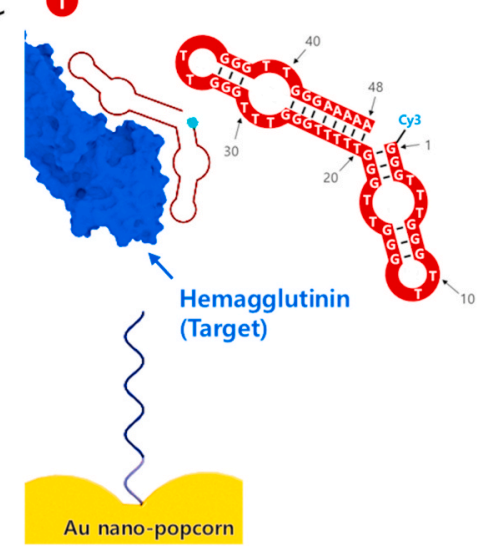

(i)

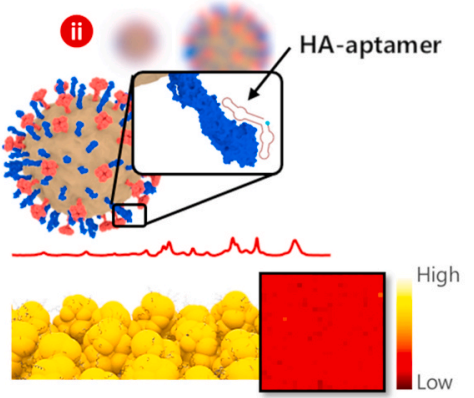

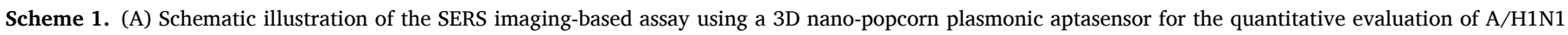

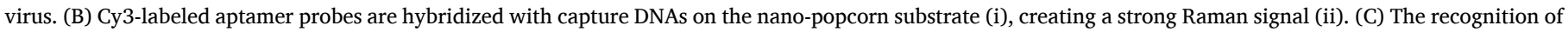
A/H1N1 virus induces a conformational aptamer change (i), leading to decreased Raman signal intensities (ii). 
A
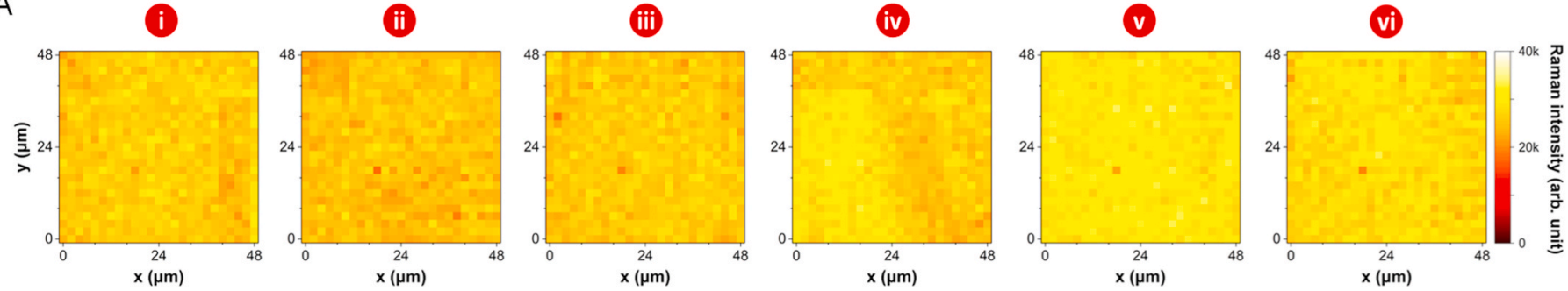

B

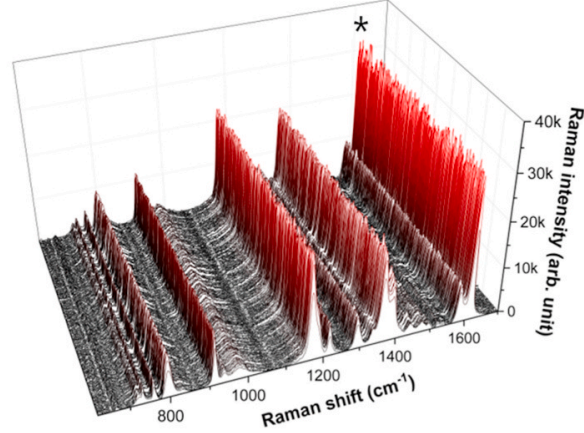

C

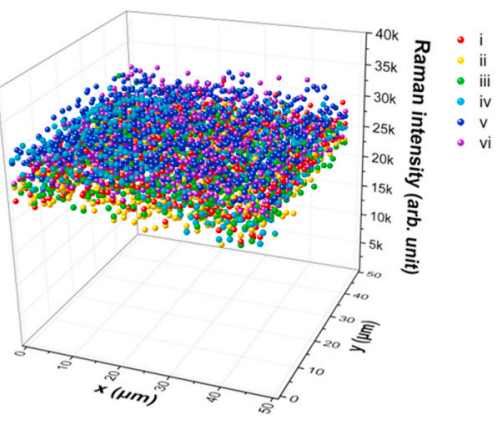

D

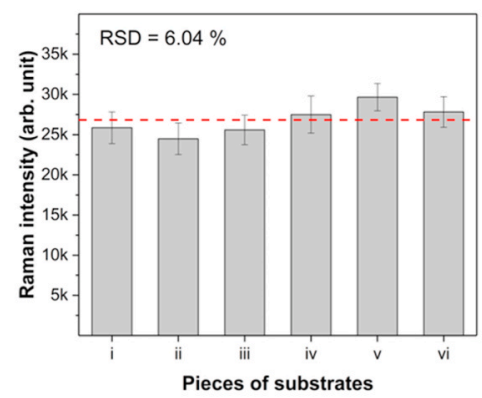

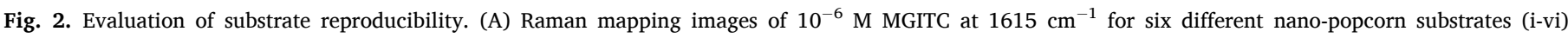

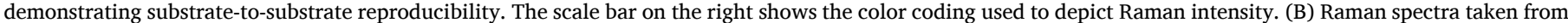

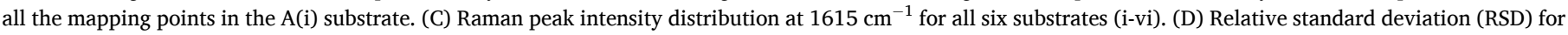
the six substrates (i-vi). (For interpretation of the references to color in this figure legend, the reader is referred to the Web version of this article.)

leading to a strong Raman signal intensity caused by the electromagnetic field enhancements. After aptamer hybridizations, the remaining gold surfaces were immediately filled with MCH. The well-organized, selfassembled monolayer formed by $\mathrm{MCH}$ prevented the nonspecific adsorption of aptamer DNAs by blocking their access to the gold surfaces. Consequently, surface functionalization with $\mathrm{MCH}$ aids in the uniform distribution of double-strand DNAs over the substrate. Additional experiments were performed to test the temporal stability of our SERS substrate. As shown in Fig. S4, Raman mapping images of Cy3 on 3D Au nano-popcorn substrates were daily measured for eight days. The uniform light-yellow distributions for all substrates demonstrate a consistent day-to-day reproducibility. According to our statistical analysis, the relative standard deviation (RSD) for six substrates was as low as $2.06 \%$ (Fig. S4C); this low RSD value indicates that the day-to-day variation in SERS intensity was very low. Based on these experimental data, it could be concluded that Au nano-popcorn substrates exhibit a very good temporal stability. In the presence of the target $\mathrm{A} / \mathrm{H} 1 \mathrm{~N} 1$ viruses, however, aptamer DNAs were released from the 3D nano-popcorn substrate surface then bound with target $\mathrm{A} / \mathrm{H} 1 \mathrm{~N} 1$ viruses by folding into unique G-quadruplex structures (Scheme 1; Shratori et al., 2014).

Fig. 3A demonstrates the Raman mapping images of 3D Au nanopopcorn substrates measured under three different sample conditions.
A 1
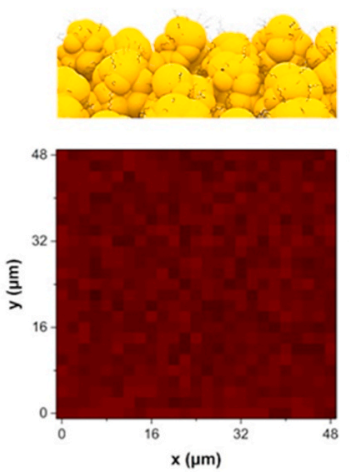
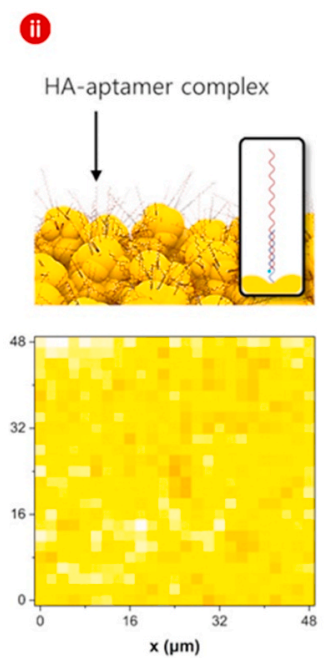
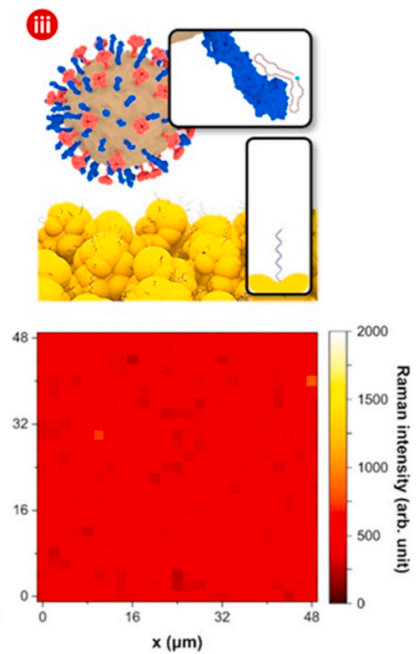

B

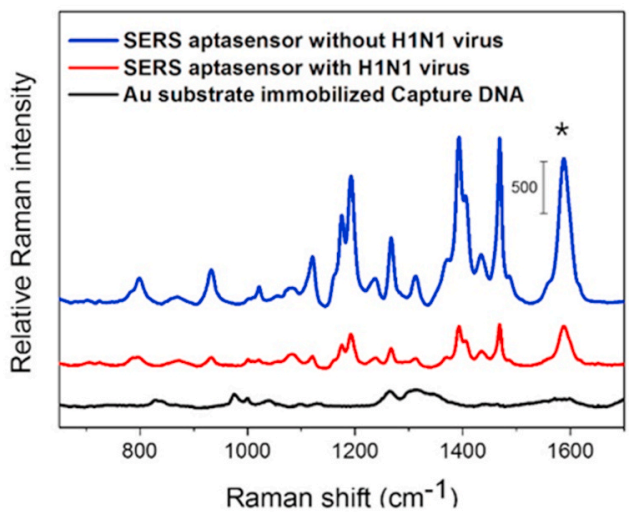

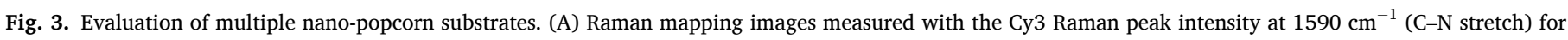

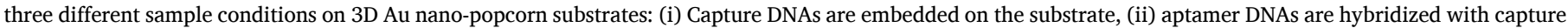

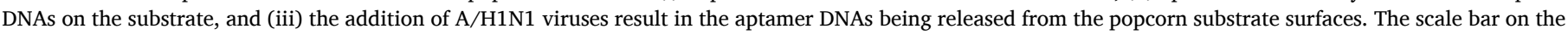

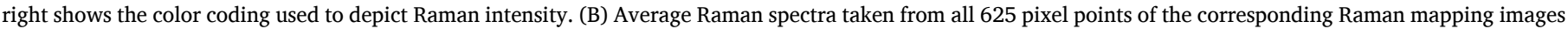
of (i), (ii), and (iii). (For interpretation of the references to color in this figure legend, the reader is referred to the Web version of this article.) 
The Raman image for capture DNA-embedded nano-popcorn substrates showed very weak Raman signal intensity distributions ( $i$, dark color) of capture DNAs over the substrates. When aptamer DNAs were hybridized with capture DNAs, Raman signal distributions were greatly enhanced (ii, light yellow color) by the Cy3 reporters at the $5^{\prime}$-end of aptamer DNAs. When $750 \mathrm{PFU} \mathrm{mL}{ }^{-1}$ of A/H1N1 viruses were added, the signal distributions decreased significantly (iii, orange color) since the aptamer DNAs were released from the nano-popcorn substrate surfaces. The Raman images shown in Fig. 3B demonstrate that the intensity distribution changes of Raman signals along the virus concentration gradient could be used for the quantitative evaluation of the A/H1N1 virus. To optimize assay time, Raman signal intensity variations for two different concentrations of A/H1N1 virus (750 PFU mL ${ }^{-1}$ and $15000 \mathrm{PFU} \mathrm{mL}^{-1}$ ) were monitored. For both concentrations, the Raman intensity gradually decreased, and then, after approximately $4 \mathrm{~min}-5 \mathrm{~min}$, reached equilibrium states (Fig. S5). Based on these results, the optimum A/H1N1 virus assay time was $5 \mathrm{~min}$. (For interpretation of the references to color in this figure legend, the reader is referred to the Web version of this article.)

It is difficult to achieve a reliable result by averaging several detection points using SERS-based assays to quantitatively analyze the A/ H1N1 virus because it is not easy to control the nanogap distributions generated by nanostructures on 3D nano-popcorn substrates. Therefore, this work used a SERS imaging-based assay platform for a reproducible assay of the A/H1N1 virus. Using the computer-controlled $x-y$ translational stage, 625 -pixel ( 1 pixel $=2 \mu \mathrm{m} \times 2 \mu \mathrm{m}$ ) mapping images were obtained for different concentrations of the A/H1N1 virus. It took approximately $11 \mathrm{~min}$ to obtain a SERS mapping image for each A/
H1N1 virus concentration. The Raman mapping images measured using the Cy3 Raman peak intensity at $1590 \mathrm{~cm}^{-1}$ (C-N stretch) for various A/ H1N1 virus concentrations ranging from 0 to $75000 \mathrm{PFU} \mathrm{mL}^{-1}$ showed that more aptamers were bound to target $\mathrm{A} / \mathrm{H} 1 \mathrm{~N} 1$ viruses as the virus concentration increased from 0 to $75000 \mathrm{PFU} \mathrm{mL}^{-1}$, leading to fewer Cy3-labeled aptamer DNAs remaining on the 3D popcorn substrate surface. As a result, the mapping image color changed from bright yellow to dark orange with increasing virus concentration (Fig. 4A). The average Raman spectra for 625 points of the SERS mapping pixels in the presence of various $\mathrm{H} 1 \mathrm{~N} 1$ virus concentrations showed that the Raman peak intensity at $1590 \mathrm{~cm}^{-1}$ concomitantly decreased with the increase in virus concentration (Fig. 4B). Corresponding calibration curves for H1N1 virus are displayed in Fig. 4C. Using three standard deviations from the blank, the LOD was determined to be $97 \mathrm{PFU} \mathrm{mL}{ }^{-1}$. The selectivity performance of the SERS imaging-based assay platform was also tested for two different types of influenza viruses, A/H1N1 (1500 PFU mL ${ }^{-1}$ ) and A/H3N2 (15000 PFU mL ${ }^{-1}$ ). Raman mapping images of A/H1N1, A/H3N2, and a 1:1 mixture of the two after they were added to aptamer DNA-immobilized popcorn substrates are shown in Fig. 5. When A/H3N2 virus was dropped onto the nano-popcorn biosensor, only a small change was observed in its Raman peak intensity. In contrast, the Raman peak intensity decreased significantly in the presence of $\mathrm{A} / \mathrm{H} 1 \mathrm{~N} 1$ virus. When the $1: 1$ mixture of $\mathrm{A} / \mathrm{H} 1 \mathrm{~N} 1$ and $\mathrm{A} / \mathrm{H} 3 \mathrm{~N} 2$ was introduced onto the substrate, the relative Raman peak intensity was also significantly decreased. To test the selectivity of our substrate for influenza A/H1N1 from clinical samples, we performed additional experiments with a cocktail solution mixture containing BSA $(0.1 \%=1$ $\left.\mu \mathrm{g} \mathrm{mL}^{-1}\right)$, mucin $\left(1 \mu \mathrm{g} \mathrm{mL}^{-1}\right)$, artificial serum (10\%), influenza A/H1N1

A
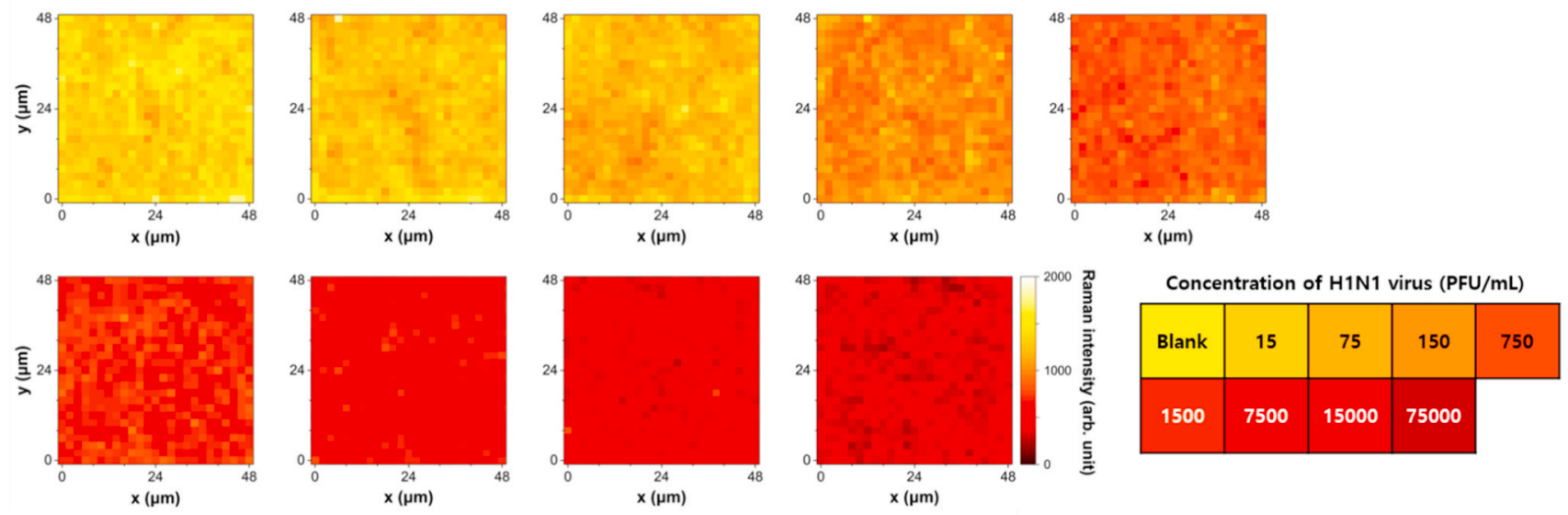

B
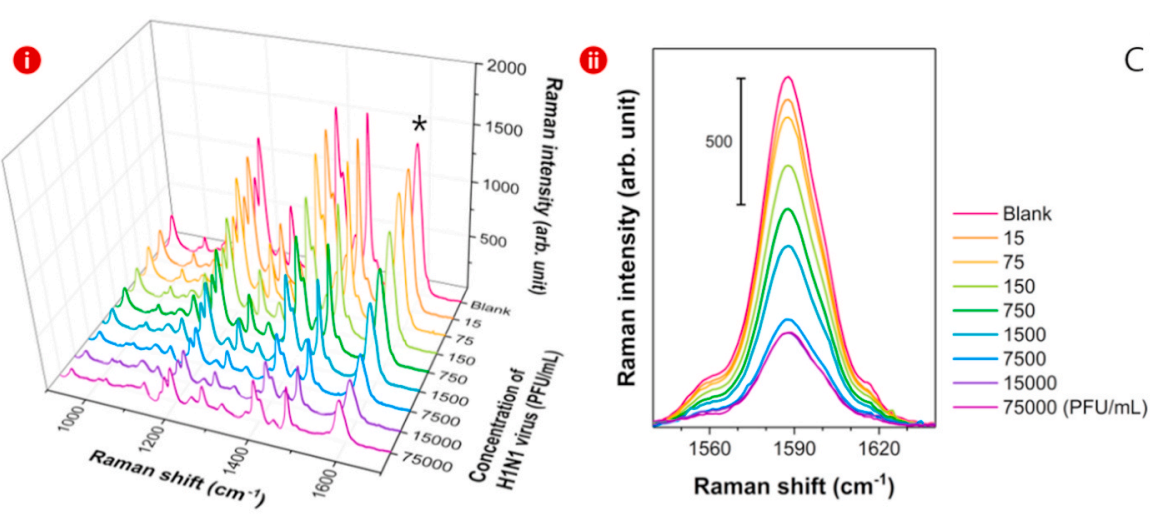

C

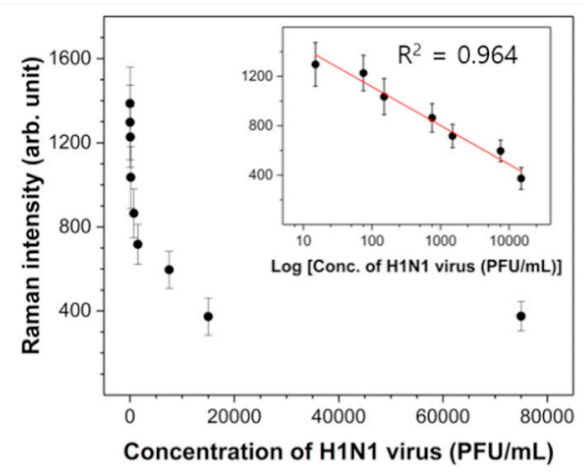

Fig. 4. Evaluation of Raman intensity variation with increasing virus concentration. (A) Raman mapping images, measured at $1590 \mathrm{~cm}^{-1}$ for various A/H1N1 virus concentrations ranging from 0 to $75000 \mathrm{PFU} \mathrm{mL} \mathrm{m}^{-1}$. The scale boxes on the right shows the color decoding box for Raman intensity. (B) Average Raman spectra for

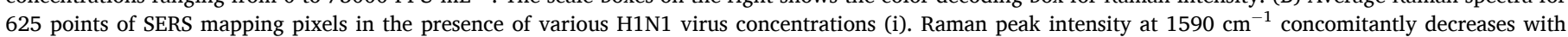
increasing virus concentrations (ii). (C) Calibration curve for various concentrations of H1N1 virus obtained from average Raman spectra. The insert shows the calibration line for the lower virus concentration ranges $\left(10-10000 \mathrm{PFU} \mathrm{mL} \mathrm{L}^{-1}\right.$. Error bars indicate the standard deviation from three measurements. (For interpretation of the references to color in this figure legend, the reader is referred to the Web version of this article.) 
A

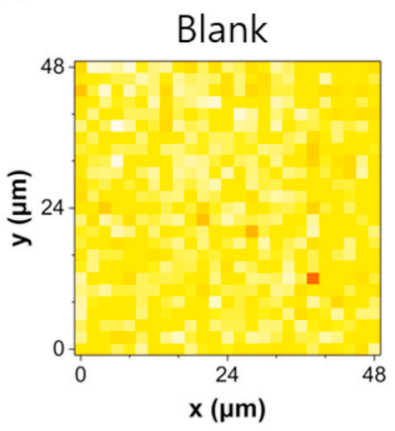

B

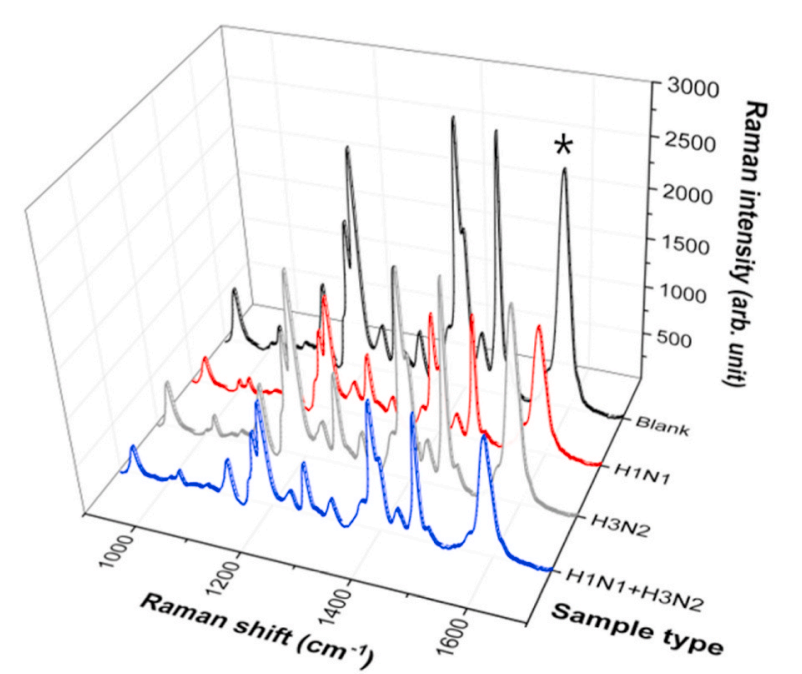

$\mathrm{H} 1 \mathrm{~N} 1$

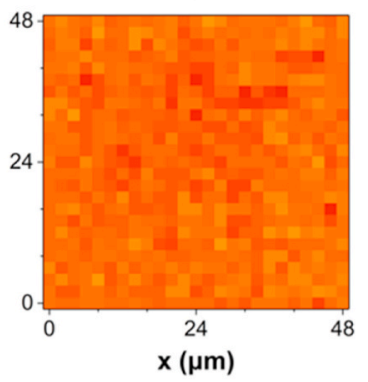

48
H3N2

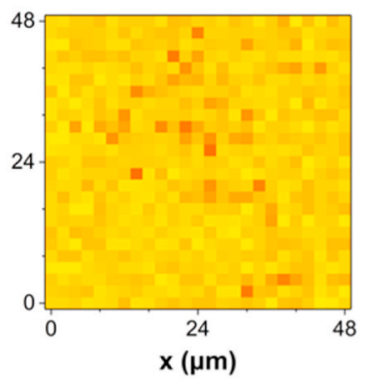

C
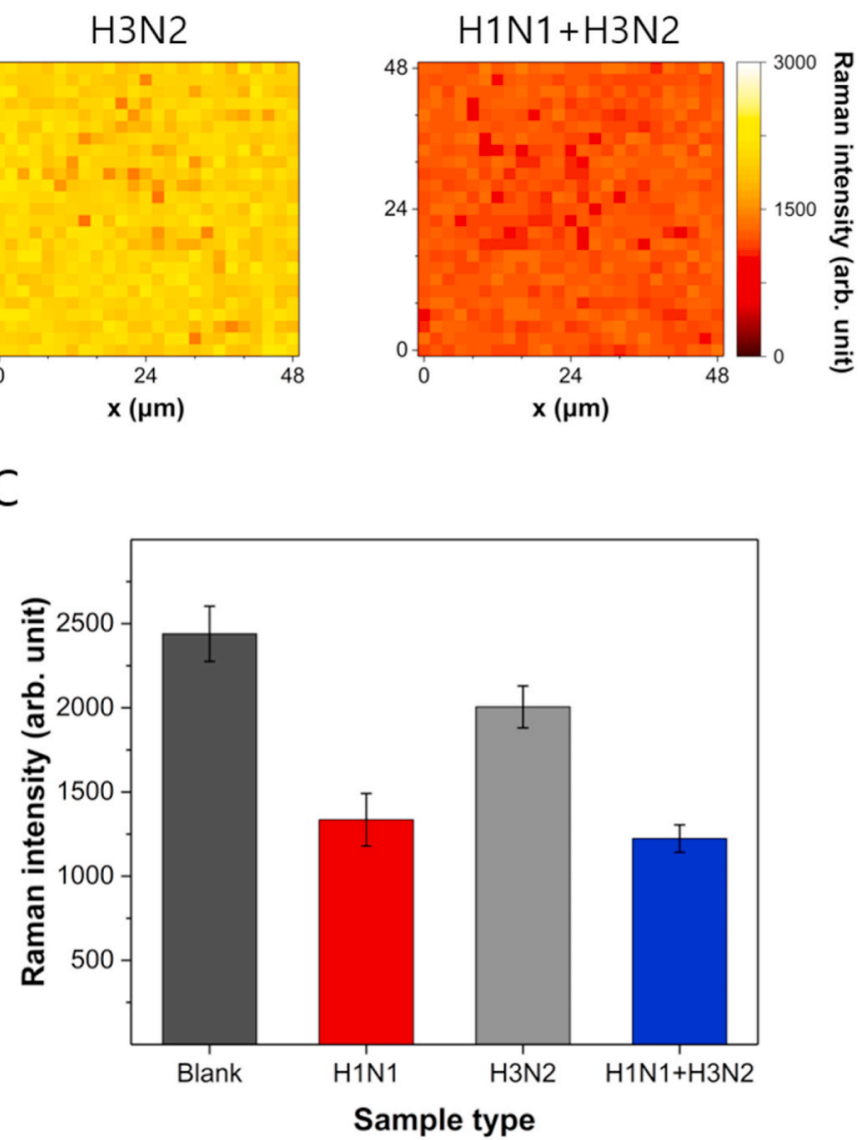

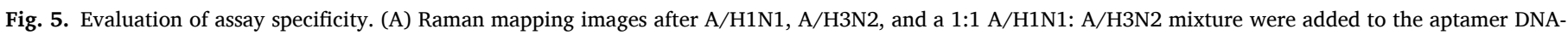

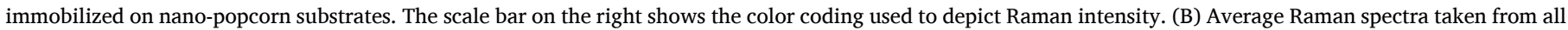

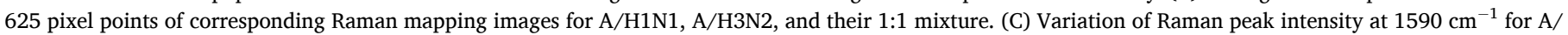

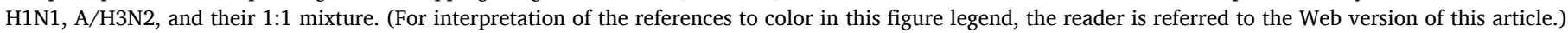

virus (7500 PFU mL ${ }^{-1} \approx 0.325 \mathrm{ng} \mathrm{mL}^{-1}$ ). Fig. S8A demonstrates the corresponding Raman mapping images of 3D Au nano-popcorn substrates measured under six different sample conditions. As shown in Fig. S8B, BSA, mucin and serum do not take a serious effect on the quantitative analysis of influenza A/H1N1 virus. These results indicated that our 3D nano-popcorn aptasensor could be successfully utilized as a diagnostic platform for the classification of different types of influenza A virus. When the LOD of the current sensor was compared with that described in previous studies (Table S1), the LOD of our SERS imagingbased assay was more sensitive by approximately one or two orders of magnitude. Moreover, our approach consumed a small sample volume $(3 \mu \mathrm{L})$ and the total assay time for one sample was estimated to be 20 $\min$.

\subsection{Performance evaluation of SERS imaging-based assay platforms}

To estimate the LOD and reproducibility of the proposed SERS imaging-based assay platform, ELISA assays for various concentrations of $\mathrm{A} / \mathrm{H} 1 \mathrm{~N} 1$ virus were performed and the results were compared with those obtained from our SERS-based aptasensor. Fig. S6 displays the colorimetric ELISA assay results and corresponding calibration curve for various concentrations of $\mathrm{A} / \mathrm{H} 1 \mathrm{~N} 1$ virus from 0 to $10^{8} \mathrm{PFU} \mathrm{mL}^{-1}$. The enzymatic catalytic reactions induced a color change from colorless to dark yellow with increasing virus concentration. The LOD of the ELISA tests was estimated to be $15600 \mathrm{PFU} \mathrm{mL}{ }^{-1}$. Therefore, the detection sensitivity of our SERS-based aptasensor for detecting the A/H1N1 virus was approximately three orders of magnitude higher than that of ELISA.
Additionally, ELISA experiments using aptamers was also performed for the direct comparison with our SERS-based assay results. The protocol was specifically described in the supporting data. Assays were carried out in triplicate, and the calibration curve was determined. Here, a four parameter logistic fitting model has been used to obtain the fitting parameters. The fitting parameters and corresponding calibration curves for ELISA are displayed in Fig. S7. The LOD, determined by ELISA, was estimated to be $3315 \mathrm{PFU} \mathrm{mL}^{-1}$. As described above, the LOD of our

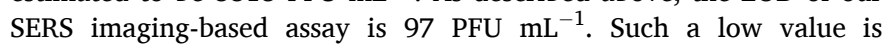
approximately 35 times more sensitive than that of the corresponding ELISA-based method using aptamers.

\section{Conclusions}

A 3D nano-popcorn plasmonic substrate was fabricated using a thermal evaporation method to develop a SERS-based aptasensor for the highly sensitive and reproducible detection of influenza A/H1N1 virus. The surface energy difference between the PFDT spacer and Au layer led to a highly uniform Au nanoparticle self-assembly and neighboring nanoparticles created multiple hotspots on the substrate. Consequently, the incident field was dramatically enhanced at hotspots by localized surface plasmon effects; the homogeneous distribution of multiple hotspots on the nano-popcorn substrate enabled reproducible target molecule analysis. When the target $\mathrm{A} / \mathrm{H} 1 \mathrm{~N} 1$ virus was added onto the substrate, the coupling between aptamer DNA and the virus decreased the Raman signal intensities. Such variation was used for the highly accurate quantitative evaluation of $\mathrm{A} / \mathrm{H} 1 \mathrm{~N} 1$ virus. The LOD and assay 
time of our SERS-based aptasensor using a 3D nano-popcorn substrate for detecting the $\mathrm{A} / \mathrm{H} 1 \mathrm{~N} 1$ virus were estimated to be $97 \mathrm{PFU} \mathrm{mL}{ }^{-1}$ and 20 min, respectively. Comparison with other platforms showed that this approach not only provides a facile diagnostic tool for influenza virus but could serve as a new reliable assay platform for other viral pathogens.

\section{CRediT authorship contribution statement}

Hao Chen: Conceptualization, Validation, Formal analysis, Investigation. Sung-Gyu Park: Funding acquisition, Methodology, Investigation, Writing - review \& editing. Namhyun Choi: Conceptualization, Methodology, Visualization. Joung-Il Moon: Validation, Formal analysis, Investigation. Hajun Dang: Validation, Investigation. Anupam Das: Validation, Formal analysis. Seunghun Lee: Validation, Investigation. Do-Geun Kim: Validation, Formal analysis. Lingxin Chen: Conceptualization, Methodology, Writing - review \& editing. Jaebum Choo: Funding acquisition, Conceptualization, Methodology, Writing review \& editing.

\section{Declaration of competing interest}

The authors declare that they have no known competing financial interests or personal relationships that could have appeared to influence the work reported in this paper.

\section{Acknowledgements}

This work was supported by the government-wide R\&D Fund for the research of infectious diseases in Korea [grant number HG18C0062] and the National Research Foundation of Korea [grant numbers 2019R1A2C3004375 and 2017M2A2A6A01019037]. This work was also supported by the Ministry of Science, ICT, and Future Planning [grant number POC3390] and the Fundamental Research Program [PNK 6800] of the Korean Institute of Materials Science [KIMS] and the Ministry of Trade, Industry, and Energy [grant number N0002310].

\section{Appendix B. Supplementary data}

Supplementary data to this article can be found online at https://doi. org/10.1016/j.bios.2020.112496.

\section{References}

Blut, A., 2009. Transfus. Med. Hemotherapy 36, 32-39. Böhm, C., Perrin, J., 1993. Rev. Sci. Instrum. 64, 31-44.

Carr, M.J., Gunson, R., Maclean, A., Coughlan, S., Fitzgerald, M., Scully, M.,

O'Herlihy, B., Ryan, J., O'Flanagan, D., Connell, J., Carman, W.F., Hall, W.W., 2009. J. Clin. Virol. 45, 196-199.

Catala, C., Mir-Simon, B., Feng, X., Cardozo, C., Pazos-Perez, N., Pazos, E., Gómez-de Pedro, S., Guerrini, L., Soriano, A., Vila, J., Marco, F., Garcia-Rico, E., AlvarezPuebla, R.A., 2016. Adv. Mater. Tech. 1, 1600163.
Chartrand, C., Leeflang, M.M., Minion, J., Brewer, T., Pai, M., 2012. Ann. Intern. Med. 156, 500-511.

Chen, C., Zou, Z., Chen, L., Ji, X., He, Z., 2016. Nanotechnology 27, 435102.

Chung, E., Jeon, J., Yu, J., Lee, C., Choo, J., 2015. Biosens. Bioelectron. 64, 560-565.

Dawood, F.S., Jain, S., Finelli, L., Shaw, M.W., Lindstorm, S., Garten, R.J., Gubareva, L. V., Xu, X., Bridges, C.B., Uyeki, T.M., 2009. N. Engl. J. Med. 360, 2605-2615.

Hasegawa, H., Savory, N., Abe, K., Ikebukuro, K., 2016. Molecules 21, 421.

Hawkes, M., Richardson, S.E., Ipp, M., Schuh, S., Adachi, D., Tran, D., 2010. Pediatrics $125,639-644$.

Jiang, Z.Y., Jiang, X.X., Su, S., Wei, X.P., Lee, S.T., He, Y., 2012. Appl. Phys. Lett. 100, 203104.

Ko, J., Park, S.G., Lee, S., Wang, X., Mun, C., Kim, S., Kim, D.H., Choo, J., 2018. ACS Appl. Mater. Interfaces 10, 6831-6840.

Ku, T.H., Zhang, T., Luo, H., Yen, T.M., Chen, P.W., Han, Y., Lo, Y.H., 2015. Sensors 15, 16281-16313.

Lee, M., Lee, S., Lee, J.H., Lim, H.W., Seong, G.H., Lee, E.K., Chang, S.I., Oh, C.H., Choo, J., 2010. Biosens. Bioelectron. 26, 2135-2141.

Lee, S., Chon, H., Lee, J., Ko, J., Chung, B.H., Lim, D.W., Choo, J., 2014a. Biosens. Bioelectron. 51, 238-243.

Lee, S., Byun, E.Y., Kim, J.K., Kim, D.G., 2014b. Curr. Appl. Phys. 14, 180-182.

Lim, E.K., Guk, K., Kim, H., Chung, B.H., Jung, J., 2016. Chem. Commun. 52, 175-178.

Merckx, J., Wali, R., Schiller, I., Caya, C., Gore, G.C., Chartrand, C., Dendukuri, N., Papenburg, J., 2017. Ann. Intern. Med. 167, 394-409.

Panning, M., Eickmann, M., Landt, O., Monazahian, M., Olschläger, S., Baumgarte, S., Reischl, U., Wenzel, J.J., Niller, H.H., Günther, S., Hollmann, B., Huzly, D., Drexler, J.F., Helmer, A., Becker, S., Matz, B., Eis-Hübinger, A., Drosten, C., 2012. Euro Surveill. 14, 1-6.

Park, S.G., Xiao, X., Min, J., Mun, C.W., Jung, H.S., Giannini, V., Weissleder, R., Maier, S. A., Im, H., Kim, D.H., 2019. Adv. Funct. Mater. 29, 1904257.

Rewar, S., Mirdha, D., Rewar, P., 2015. Ann. Glob. Health 81, 645-653.

Ruscito, A., DeRosa, M.C., 2016. Front. Chem. 4, 14.

Shanmukh, S., Jones, L., Driskell, J., Zhao, Y., Dluhy, R., Tripp, R.A., 2006. Nano Lett. 6, 2630-2636.

Shao, F., Lu, Z., Liu, C., Han, H., Chen, K., Li, W., He, Q., Peng, H., Chen, J., 2014. ACS Appl. Mater. Interfaces 6, 6281-6289.

Shratori, I., Akitomi, J., Boltz, D.A., Horii, K., Fruichi, M., Waga, I., 2014. Biochem. Biophys. Res. Commun. 443, 37-41.

Sun, Y., Xu, L., Zhang, F., Song, Z., Hu, Y., Ji, Y., Shen, J., Li, B., Lu, H., Yang, H., 2017. Biosens. Bioelectron. 89, 906-912.

Tabakman, S.M., Lau, L., Robinson, J.T., Price, J., Sherlock, S.P., Wang, H., Zhang, B. Chen, Z., Tangsombatvisit, S., Jarrell, J.A., Utz, P.J., Dai, H., 2011. Nat. Commun. 2 , 466.

Tao, A., Kim, F., Hess, C., Golfberger, J., He, R., Sun, Y., Xia, Y., Yang, P., 2003. Nano Lett. 3, 1229-1233.

Vos, L.M., Bruning, A.H.L., Reitsma, J.B., Schuurman, R., Riezebos-Brilman, A., Hoepelman, A.I.M., Oosterheert, J.J., 2019. Clin. Infect. Dis. 69, 1243-1253.

Wang, X., Park, S.G., Ko, J., Xiao, X., Giannini, V., Maier, S.A., Kim, D.H., Choo, J., 2018. Small 14, 1801623.

Whiley, D.M., Bialasiewicz, S., Bletchly, C., Faux, C.E., Harrower, B., Gould, A.R., Lambert, S.B., Nimmo, G.R., Nissen, M.D., Sloots, T.P., 2009. J. Clin. Virol. 45, 203-204.

Willets, K.A., Van Duyne, R.P., 2007. Annu. Rev. Phys. Chem. 58, 267-297.

Wu, Y., Choi, N., Chen, H., Dang, H., Chen, L., Choo, J., 2020. Anal. Chem. 92, 2628-2634.

Xu, S., Ouyang, W., Xie, P., Lin, Y., Qiu, B., Lin, Z., Chen, G., Guo, L., 2017. Anal. Chem. 89, 1617-1623.

Yang, J., Palla, M., Bosco, F.G., Rindzevicius, T., Alstrøm, T.S., Schmidt, M.S., Boisen, A., Ju, J., Lin, Q., 2013. ACS Nano 7, 5350-5359.

Yi, S.Y., Lee, U.J., Chung, B.H., Jung, J., 2015. Chem. Commun. 51, 8865-8867.

Zhang, X., Dhawane, A.N., Sweeney, J., He, Y., Vasireddi, M., Iyer, S.S., 2015. Angew. Chem. Int. Ed. 54, 5929-5932.

Zhang, Y., Lai, B.S., Juhas, M., 2019. Molecules 24, 941.

Zhou, C.H., Zhao, J., Pang, D.W., Zhang, Z.L., 2014. Anal. Chem. 86, 2752-2759.

Zhu, C., Meng, G., Zheng, P., Huang, Q., Li, Z., Hu, X., Wang, X., Huang, Z., Li, F., Wu, N., 2016. Adv. Mater. 28, 4871-4876. 